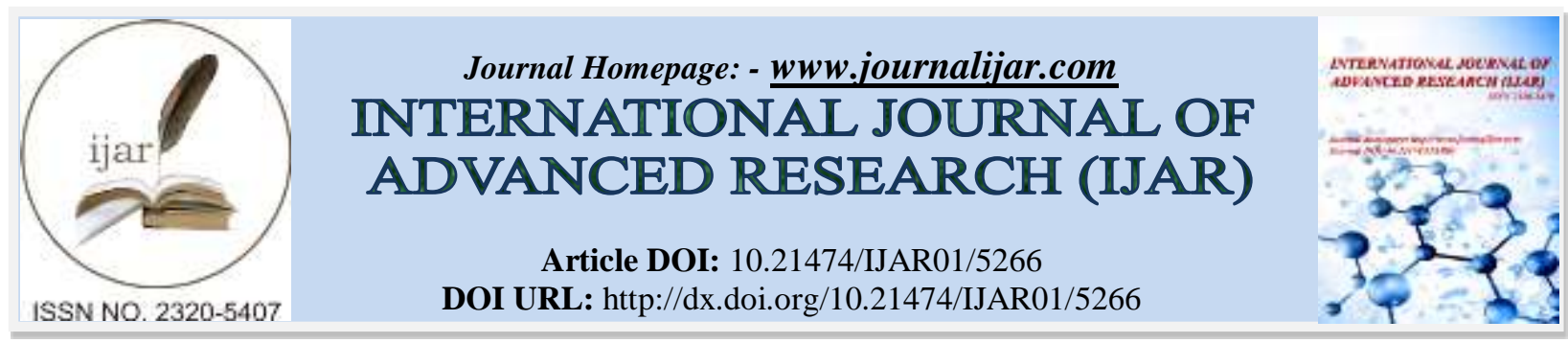

RESEARCH ARTICLE

\title{
QUANTUM PLASMA CONDENSATE AS A NEW SOURCE OF ELECTRIC ENERGY. MHD- GENERATOR WITH PLASMA SEPARATION.
}

\begin{abstract}
A. V. Kulakov.
Expert-analytical Centre of the Ministry of Education and Science of the Russian Federation, Moscow, Russia.
\end{abstract}

\section{Manuscript Info}

Manuscript History

Received: 24 June 2017

Final Accepted: 26 July 2017

Published: August 2017

\section{Key words:-}

new alternative environmentally safe source of electric energy, non-ideal plasma, new state of matter-quantum plasma condensate, energy conversion, power release mechanism, physical and chemical methods of power generation, purification of the ecosphere of the planet.

\begin{abstract}
For the first time in history it has been theoretically predicted and confirmed that there exists a new state of matter -quantum plasma condensate combining properties of normal liquid and ionized plasma. This paper explores the quantum nonideality of nondegenerate plasma and exchange interaction of particles in it leading to efficient energy release in the plasma process under consideration. It looks at the data on the methods developed by the author for converting quantum plasma condensate energy to heat, uv and x-ray radiation, energy of generation and acceleration of charged particles. Special attention is paid to one of the most demanded application of quantum non-ideal plasma: electric energy generation. The author has proposed and investigated a revolutionary device for $\mathrm{dc}$ and ac electric energy generation based on using quantum plasma condensate - mhdgenerator with plasma separation, being the only environmentally safe source of electric energy.
\end{abstract}

Copy Right, IJAR, 2017,. All rights reserved.

\section{Introduction:-}

Creating energy nature-like technologies that do not cause damage to the environment and help restore the disrupted balance between the biosphere and technosphere is one of the most important in the world.

Indeed, with the preservation of the existing industrial technologies the world's resources are increasingly depleted, mankind has nothing to pay for purification and restoration of environment. Only new technologies and resources will save humanity from an imminent collapse. The way out consists in finding a new alternative renewable harmless and sustainable source of energy, the very use of which is a whole industry for processing and disposing of accumulated wastes and the recycling of which is only comparable to that of the whole transport and energy complex. In this case, the present level of consumption in the world can be preserved helping to implement the concept of sustainable growth.

The solution for the above problem is spelled out in this paper.

The state of liquefied plasma (quantum plasma condensate) that we have discovered is an entirely new state of matter combining features of a normal liquid (liquidity, surface tension, and internal correlations) and ionized plasma in the usual sense. Theoretical concepts of such a state are based on the quantum theory of exchange forces in condensed media $[1,2,6]$. The essential feature of such forces is their collective (npnpaired0 nature which 
ultimately determines the long-range ordering in interatomic interactions. Generally, molecular forces and especially forces that determine long-range ordering are of a purely quantum nature. The main idea is that electronic shells in liquefied plasma (e.g., discharge plasma) overlap, and this overlap of interline nature, i.e., overlapping with diffraction peaks and drops of the wave function, nevertheless, effectively leads to formation of quantum attractive forces between discharge ions.

Quantum forces are a natural fact since it has been repeatedly proven by experiments conducted by research scientists who deal with discharges. [3,4]Exchange interaction of electrons under such conditions leads to the attraction of ions to each other, whereby the bond energy of the former becomes negative. Such states often occur spontaneously in natural conditions. Quantum forces are determined by a sequential overlapping of electronic shells belonging to the adjacent atoms or ions. Such overlapping creates a first-order effect in relation to de Broglie wave length to the distance between ions. If overlapping also exists in matter that is in a normal phase state, but decreases exponentially with the growing distance between atoms, then in plasma (since the spectrum of quantum energy states is continuous) the effect of shell overlap intensity decrease is considerably decelerated with the growing distance between atoms and is described by a power-law dependence. As a result, particles engage with each other in such a manner that correspond to a chain of sequentially overlapping electron clouds, with each branch of the chain spreading over a distance approximately equal to the screening radius. The chain covers the whole plasma. plasma ions trapped by this chain are attracted to each other, which causes plasma phase transformation to occur. Conversion to a new state is accompanies by the release of energy equal to transformation heat.

The fact that overlapping of electron shells leads to their effective engagement is well known from the chemical bond theory. We shall indicate the classical theory of molecular force of Heitler-London, in which such forces are found in calculating the simplest molecules based on variations. Variation methods in physics are considered as intuitive, a posteriori methods. Only a theory based on the direct solution of the fundamental equation of quantum physics - the Schrodinger equation. can be considered as a consistent heuristical one. Covering the class of states of continuous spectrum that is expressed by a relation of electron states in plasma, the perturbation theory considering exchange forces (Pauli principle) helped explain the peculiarities of plasma phase that have been observed as well as predict those properties of this phase which may and must be used by modern science and technology $1,2,6]$.

In modern plasma physics virtually all research efforts are focused in the field of high temperature plasma. However the focus is on "hot" fusion, the difficulties in implementing it in Earth conditions are well known. In the meantime, there exist plasma phase sources of energy determined by the collective nature of interactions of particles, which most clearly manifest themselves in sufficiently dense plasma (with a particle concentration of $\mathrm{n}=$ $10^{19}-10^{21} \mathrm{~cm}^{-3}$ ) in the low temperature region [1,7,8]. This plasma can be generated in a much simpler way than that designed for fusion. One of the methods for generating such plasma is compression by impulse electric discharge.

With relatively low temperatures plasma becomes non-ideal since the energy of Coulomb interaction of particles in such plasma turns out to be comparable with heat background energy. However the essential feature of such plasma, and this circumstance is the main one, consists in the fact that its state is to a great extent determined by quantum forces arising in it. As the normal classification suggests, plasma is not degenerate under such conditions, in the meantime, the average distance between electrons exceed by several times de Broglie wave length of heat electrons characterizing the quantum nature of a particle system, while the distance between atoms meets the following equation $[1,2]$ :

$\mathrm{r}<10 \lambda<\mathrm{r}_{\mathrm{d}}$

i.e. the screening radius exceeds the Debye screening radius by an order of magnitude.

Quantum forces in plasma create an effect of long-range order forces which are known to cause phase transformation in matter. An increasing plasma concentration results in a sharp increase in exchange bonding between electron0ion complexes so that plasma form some kind of condensate, which retains the degree of ionization while manifesting phase state properties typical of a liquid, i.e., phase transformation occurs, The former, as in the case with normal phase transitions in matter, is accompanied by the release of energy, which, however, turns out to be significantly greater than in normal phase processes. 
Specific energy release (calculated per gram) corresponding to the hidden heat of phase transformation is equal to the following value [1]:

$$
\mathrm{E}_{0}=10 \mathrm{z}^{3} \mathrm{e}^{2} \mathrm{n}^{1 \mid 3} / \mathrm{m}_{\mathrm{i}}
$$

where $\mathrm{e}=-4.810^{-10} \mathrm{cgs}-$ electron charge, $\mathrm{z}-$ ionization degree of atoms, $\mathrm{m}_{\mathrm{i}}-$ ion mass.

Supposing $\mathrm{n}=10^{21} \mathrm{~cm}^{-3}$, for estimating $\mathrm{z}=2, \mathrm{~m}_{\mathrm{i}}=2 \cdot 10^{-23} \mathrm{~g}$, we receive $\mathrm{E}_{0}=10^{13} \mathrm{erg} / \mathrm{g}=1 \mathrm{MJ} / \mathrm{g}$, which exceeds the energy release of the most efficient fuels (without the exception of nuclear fuel, of course).

It is highly important that energy release is not related to any atomic transformation or chemical reactions, but with formation of a specific ionized conglomerate which have properties typical of a light liquid, in particular a surface tension that enhances its resistance to decay.

The authors have developed methods for converting quantum plasma condensate energy to heat[7], UV and X-ray radiation[8], energy of generation and acceleration of charged particles.[5,6]

This paper offers a revolutionary technically new device which converts almost all kinetic energy of plasma to electric energy. This device uses plasma phase separation into two components - normal, so to say, vaporous component and liquid component with sufficient density and surface tension. This component that has the properties of liquid gas triggers a circulation mechanism of transferring energy to the generator and helps remove a number of significant drawbacks which today prevent MHD-device from using $[\mathbf{9 , 1 0}$. If implemented in a timely manner, the principal side of the proposed scheme will help realize plasma superlow circulation generation which would be a genuine break-through in power generation.

The flow sheet is shown in the attached figure. Gas or weakly ionized plasma is compressed by a piston A, a pulsed magnetic field can be used as such. As plasma is compressed, it is heated and delivered over power lines configured as shown in the Figure to a narrow neck B. Using the condition of plasma energy flow conservation, $\boldsymbol{\rho} \boldsymbol{u}^{3} \boldsymbol{S}=$ const, where $\boldsymbol{\rho}=\boldsymbol{m} \boldsymbol{n}_{\mathbf{0}}, \boldsymbol{n}_{\mathbf{0}}$ - density, u - plasma speed, $\boldsymbol{S}=\boldsymbol{R} \cdot \boldsymbol{a}$, a - plasma layer width.

Thus, plasma temperature increases in the equation $\boldsymbol{R}^{-2 / 3}$. Assuming, for instance, that $\boldsymbol{a}=\boldsymbol{R}_{A}=\mathbf{3 0} \mathbf{c m} ; \boldsymbol{R}_{\boldsymbol{B}}=$ $3 \mathbf{~ m m}$, then temperature increase will be 20 -fold. With initial temperature $\sim 500^{\circ} \mathrm{C}$, we obtain that the temperature in the neck will turn out to be $10-15$ thousand degrees. The ionization originating from valency shells of atoms is almost complete.

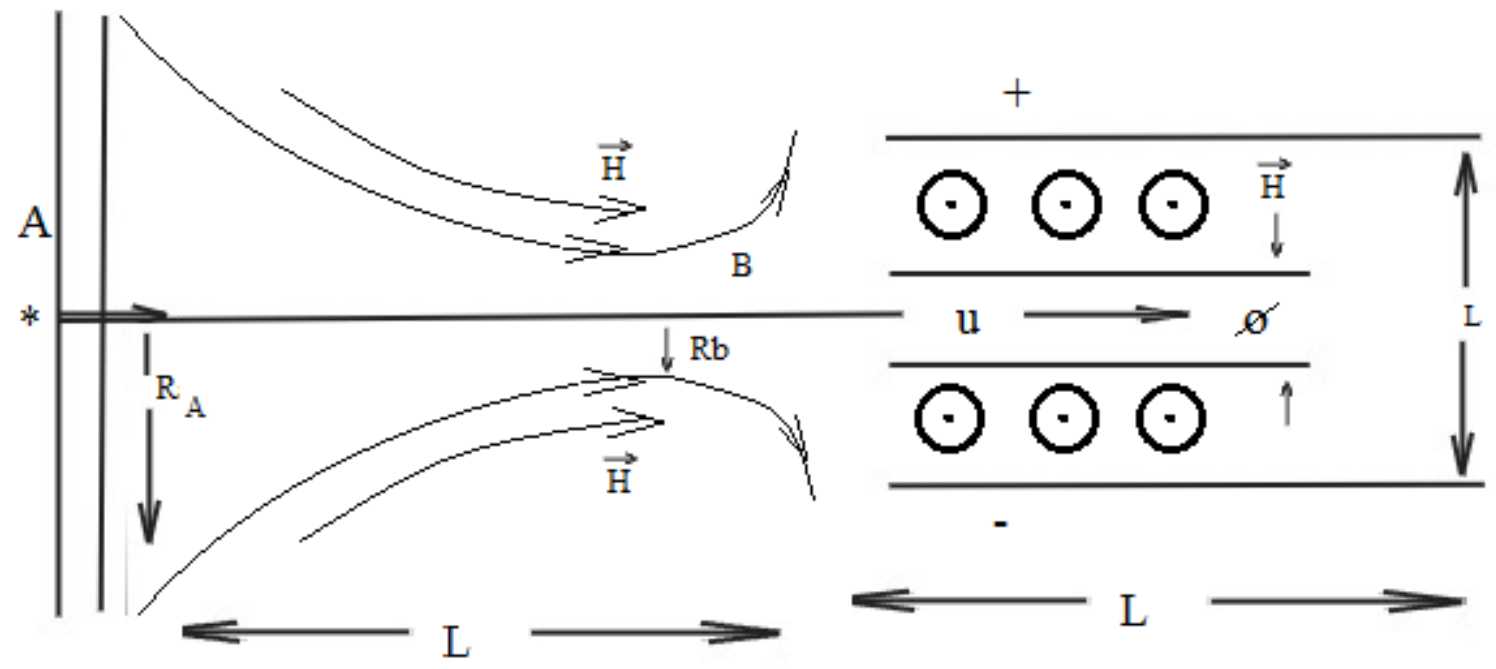

Fig. 1:- Plasma Phase Separation MHD-Generator Diagram

Under such temperatures, in accordance with the analysis that we have repeatedly carried out, phase transformation occurs if the concentration of atoms is $\boldsymbol{n}_{\mathbf{0}} \sim \mathbf{1 0}^{\mathbf{1 9}} \div \mathbf{1 0}^{\mathbf{2 0}} \mathbf{c m}^{-3}$. 
The liquid plasma layer with thickness $\boldsymbol{R}_{\boldsymbol{B}}$ and width a enters the gap between the armature, of the condenser which is basically a MHD-generator in this case (in which points are used to indicate the direction of external magnetic field). The steam created by liquid plasma is between the dense plasma jet and armature of the condenser. This steam must have a temperature that is considerably lower than plasma temperature since its generation is associated with the energy that particles spend to overcome the binding forces acting inside the plasms.

The application of a liquid plasma phase in the device under consideration allows the cyclic diagram to be used (Fig.2). Plasma belt circulates in a channel created by a suitably selected field configuration; here plasma diamagnetism is used as well as its ability to hold its shape, which is typical of liquid phase in general. There appears a picture of a plasma conveyor, whose "belt" moves with speed $\mathrm{u}$, whereby the generation of an electric field occurs as the "belt" passes inside the condenser. The energy of rotating plasma is gradually passed on to the electric field in the condenser. It is not hard to see that the number of revolutions that plasms will make before transferring all of its energy to the MHD-source is equal to:

$$
\mathrm{V}=\left(\frac{C}{U_{A}}\right)^{2} \frac{\Delta}{L} ; U_{A}=\frac{H}{\sqrt{u \pi \rho}} \ll c
$$
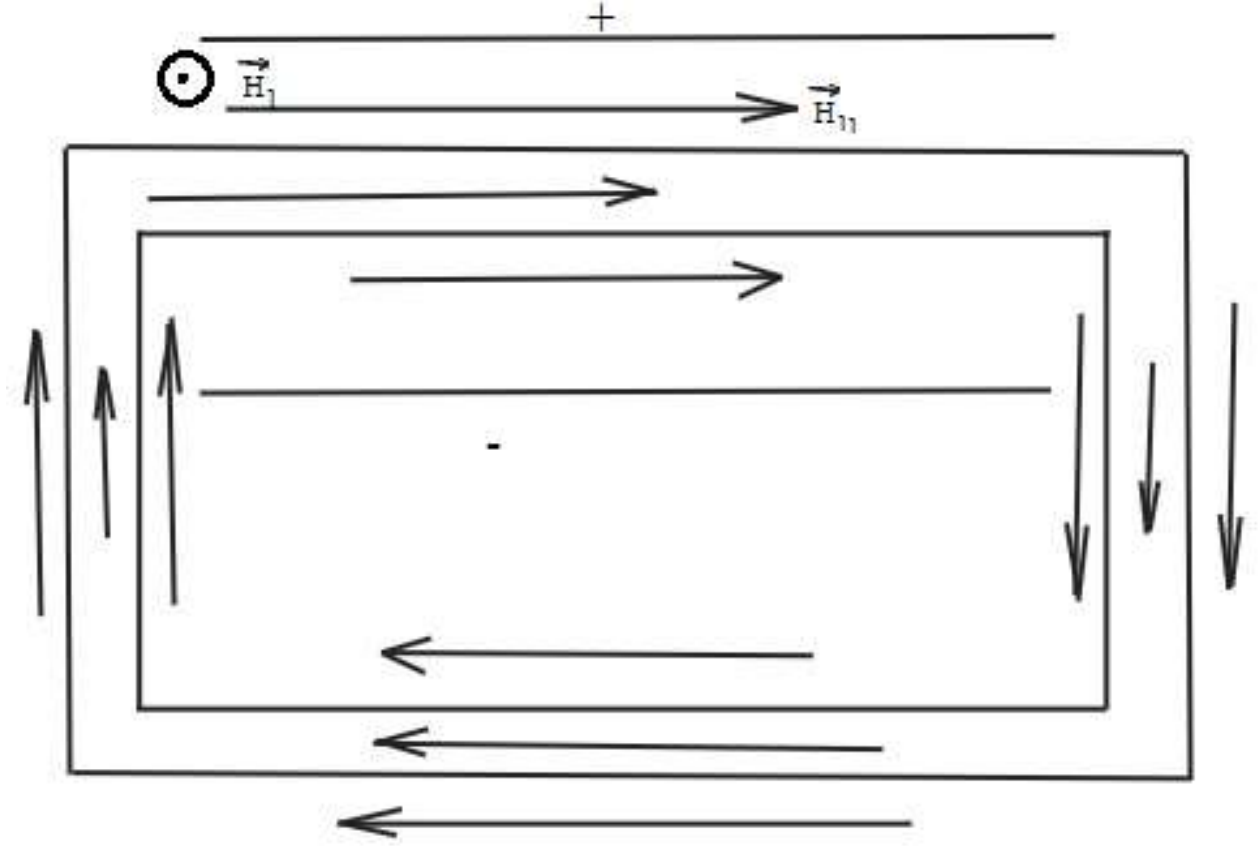

Fig. 2:- Plasma Liquid Phase Circulation Diagram. Double arrows show power lines of the master magnetic field $\left(\boldsymbol{H}_{\mathbf{1 1}}\right)$. Condenser plates are marked with plus and minus signs.

In conducting energy analysis of MHD-device operation, care should be taken to identify and explore three stages. At the first stage the above transformation takes place leading to energy release, we will identify the value of this release as $\boldsymbol{E}_{\mathbf{1}}$. This energy is tramsferred to plasma "vapour". At the second stage the layer enters the MHDcondenser entraining the vapour, whose mass is comparable with that of the liquid layer. As the former cools down, additional energy is transferred to the vapour $\boldsymbol{E}_{2}$. The vapour comes through the condenser region and its energy can be utilized. for instance, in turbines c (efficiency factor $T$ ). The functioning plasma layer transfers virtually all of its energy to the condenser. Losses are due to evaporation which, however, are insignificant when the temperature of the layer is decreased.

Therefore, the efficiency factor of the device by the order of magnitude is equal to

$\imath=\frac{m_{c}+\eta_{n}}{m_{c}+m_{n}}$

where $\boldsymbol{m}_{\boldsymbol{c}}, \boldsymbol{m}_{\boldsymbol{n}}$ - mass of the layer and "vapour" respectively.

Assuming for assessment $\boldsymbol{m}_{\boldsymbol{c}}=\boldsymbol{m}_{\boldsymbol{n}}, \chi_{\boldsymbol{T}}=\mathbf{5 0} \%$, we obtain $\chi=\mathbf{7 5} \%$, i.e. which is perfectly optimum in the modern understanding of efficiency factor. 
Estimate the power generated in the option under consideration. By the order of magnitude this power is equal to

$$
\boldsymbol{A}=\frac{\rho U^{2} L^{2} \Delta}{\mathrm{V}_{\bar{U}}^{L}}
$$

Using (1), from here we shall find $\boldsymbol{A}=\boldsymbol{\rho} \boldsymbol{U}^{3} \boldsymbol{L}^{2}\left(\boldsymbol{U}_{\boldsymbol{A}} / \boldsymbol{c}\right)^{2} ; \boldsymbol{U}_{\boldsymbol{A}} \sim \mathbf{3} \cdot \mathbf{1 0} \frac{\mathrm{cm}}{\mathrm{c}}$ Alfven velocity (at $\boldsymbol{H} \sim \mathbf{3}$. $10^{4} \mathbf{G s}, \rho=10^{-3} \mathbf{g} / \mathbf{c m}^{3}$ ), when $A \sim 1 / 3 \mathbf{~} \mathbf{W}$ - the power and the plant itself can be regarded as demonstration.

Let us analyze the aspect of energy release.

The calculation of portions for quantum energy yield (calculated per one ion) the value

$$
\varepsilon=z^{3} e^{2} n^{1 / 3}
$$

where $\boldsymbol{z}$ - ion charge, $\boldsymbol{e}-\mathbf{4 , 8} \cdot \mathbf{1 0}^{-\mathbf{1 0}} \mathrm{cgs}, \boldsymbol{n} \mathbf{c m}^{-3}$ - ion concentration. It is beneficial to use easily ionizable gases, but such that their electron shells (in other words, atomic radii) could be long enough - this is necessary to create a quantum overlapping of shells. If the energy is $\varepsilon>\boldsymbol{z I}$, where $\boldsymbol{I}$ - ionization energy, calculated for one electron i.e.

$$
\xi=\frac{z^{2} e^{2} n^{1 / 3}}{I}>1
$$

then the process becomes energy-efficient.

For example, at $\mathbf{n 1 0}^{20} \div \mathbf{1 0}^{21} \mathrm{~cm}^{-3}, z=3 \div 4 ; I=10 \mathrm{eV}=1,6 \cdot 10^{-11} \mathrm{erg}$, the above ratio exceeds unity $\xi \sim 3$. This means that plasma of a definite composition can "burn" (just as in a chemical process) and produce "combustion" heat. Certainly, this energy comes from an unusual source: here plasma behaves as a single molecule - unitary medium, but apparently there is an effect. The resulting plasma must demonstrate the properties of a liquid, which makes it convenient to use

Energy relations (in a technical aspect) are calculated as follows:

Calculated by one nucleus (or ion) the bond energy released as a result of plasma " liquefaction" is equal to $\varepsilon=\mathbf{z}^{3} \mathbf{e}^{2} \mathbf{n}^{1 / 3} \simeq \mathbf{2 0} \cdot 10^{-19} \cdot \frac{1}{3} \cdot \mathbf{1 0}^{7}=10^{11} \mathrm{erg}$ (concentration $n \approx \mathbf{1 0}^{20} \mathrm{~m}^{3}$, or density in the discharge is $\left.\rho=20 m_{H} \cdot n^{\bullet} 10^{-2} \Gamma / \mathrm{CM}^{3}\right)$.

The speed of plasma heat leak from the gun, where "liquefaction" takes place, is equal to $\boldsymbol{u}=$ $\left(\varepsilon / 20 m_{H}\right)^{1 / 2} \sim \mathbf{1 0}^{6} \mathbf{~ c m} / \mathbf{s e c}$, but can be slightly higher, it is possible $3 \cdot \mathbf{1 0}^{6} \mathbf{~ c m} / \mathbf{s e c}$.

Over time t the mass comes out of the gun $\Delta \boldsymbol{m}=\dot{\mathrm{m}} \boldsymbol{t}=\boldsymbol{\rho} \boldsymbol{u l} \boldsymbol{\delta} \boldsymbol{t}$, where $\boldsymbol{\delta}$-plasma layer thickness, $\boldsymbol{l}$ - its width. Assume $\boldsymbol{t}=\frac{\boldsymbol{L}}{\boldsymbol{U}}$, where $\boldsymbol{L}$ - profile length of the MHD-unit. Then $\Delta \boldsymbol{m}=\boldsymbol{\rho} \boldsymbol{L l} \boldsymbol{\delta}$. Assume $\boldsymbol{l}=\mathbf{0}, \mathbf{1} \boldsymbol{L} ; \boldsymbol{\delta}=\mathbf{0}, \mathbf{0 0 1} \boldsymbol{L}=$ $\mathbf{0 , 1} \mathrm{cm} ; \boldsymbol{L}=\mathbf{1 0 0} \mathrm{cm}$, we obtain $\Delta \boldsymbol{m}=\mathbf{1}$ г mixtures (type $\mathrm{C}$, Al, Na, Cs and other light chemical elements with air). 


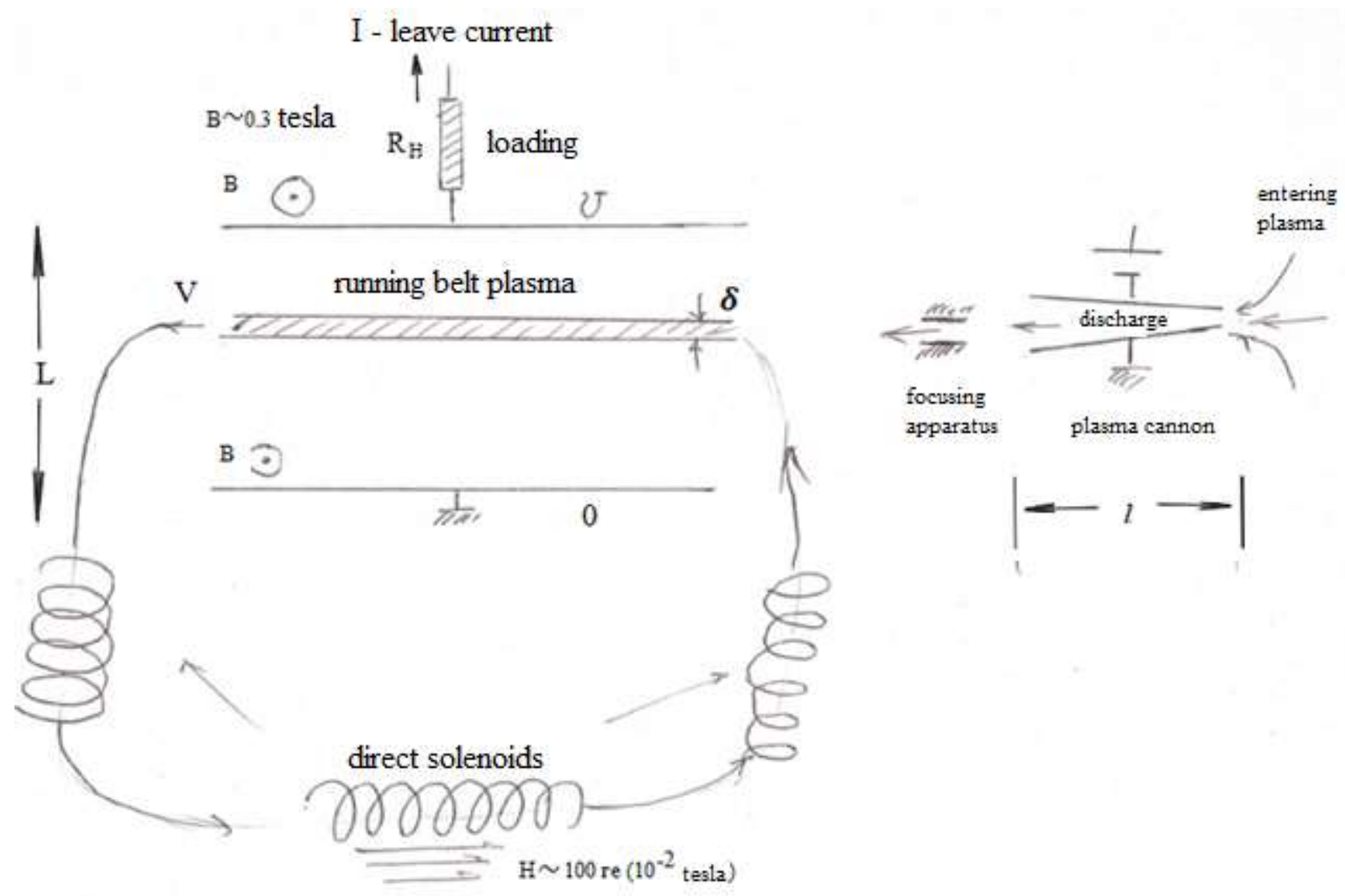

Fig.3:- The diagram of the MHD-generator on liquefied plasma

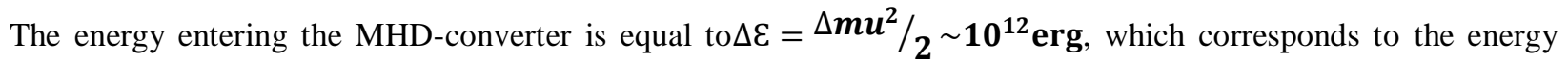
yield $\sim 100 \mathrm{~kW}$.

Entering inside the MHD-plant liquid conductive plasma creates an electric field with intensity (unipolar induction) $\overline{\boldsymbol{E}}^{\mathbf{1}}=-\frac{v}{\boldsymbol{c}} * \mathbf{B}$; by the modulus $\boldsymbol{E}=\mathbf{3 0} \mathbf{V} / \mathbf{c m}$, where the induction is $\boldsymbol{U}=\boldsymbol{E} \boldsymbol{l}=\mathbf{3 k V}$, if $\mathbf{B}=0.3$ tesla.

The closing solenoid system rotates plasma (having an abnormal magnetic moment and in accordance with the quantum magnetic dynamo). It circulates in the generator unit until it loses all excitation energy by the inductive mechanism of electric field.

The efficiency factor of magnetic transformation is known to have an order of $50 \%$, and can be increased up to $90 \%$ for the proposed diagram since plasma circulates in the magnetic field practically without leaks. $10 \%$ are determined by losses related mainly to the edge effects. Each gram with a gas mixture produces a power of $\sim 100$ $\mathrm{kW}$ in the form of permanent or alternative current with a frequency equal to plasma circulation frequency $(\sim$ $\boldsymbol{u} / \boldsymbol{L})$

Initial gas pressure is approximately $1 \mathrm{~atm}$. Ionizing a mixture of volatile elements creates plasma which, when compressed by a "piston" the function of which is performed by magnetic power lines, plasma is condensed and compressed. However such condensation is possible in the space of a discharge chamber. In this case magnetic power lines act as guideways for liquefied plasma.

The below parameters much as the diagram of the unit can slightly vary depending on specific capabilities. However in any cases the first stage is carrying out experiments in order to ascertain that plasma can really be condensed to a liquid layer. 


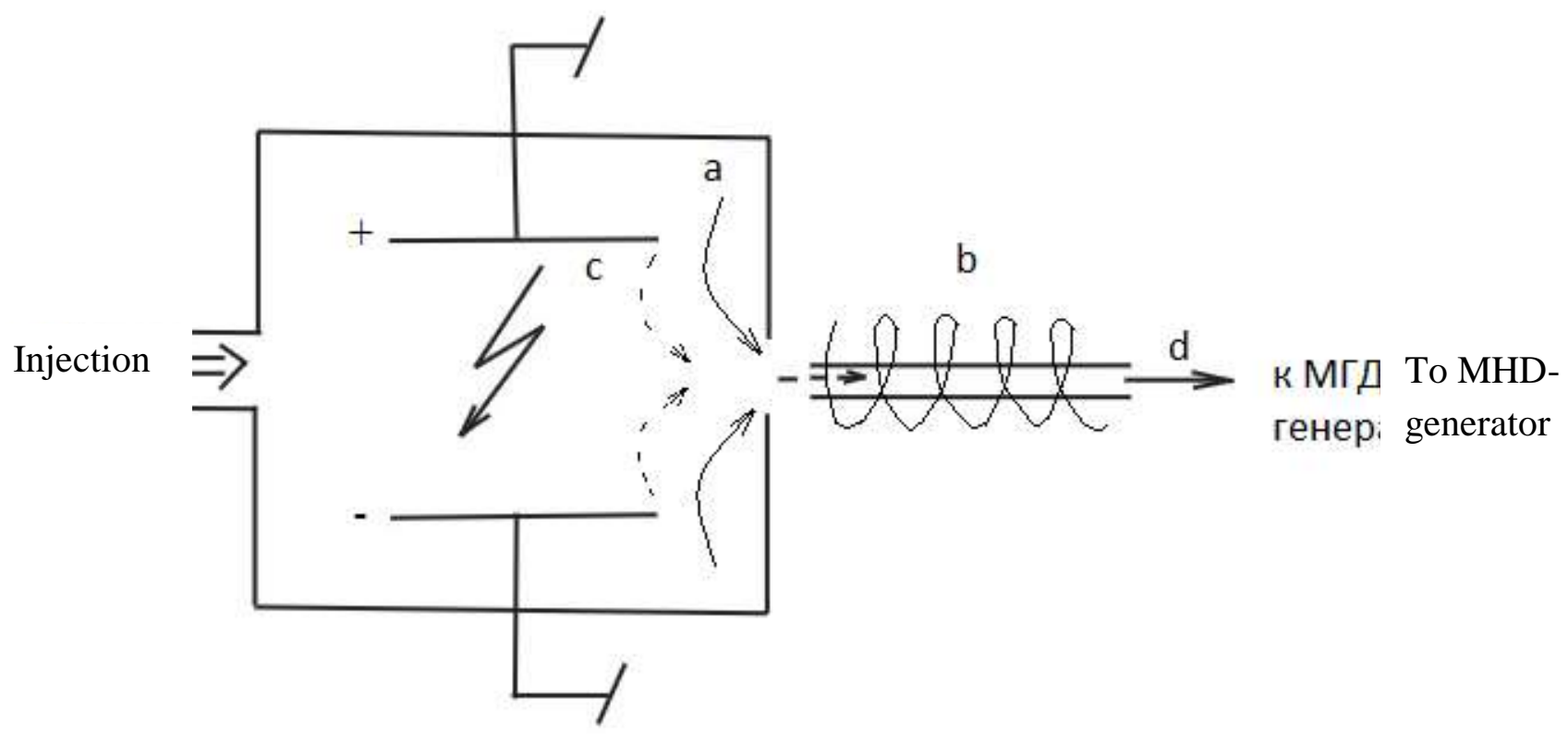

Fig.4:- The diagram of an experimental chamber for condensing plasma.

a - chamber with dimensions $30 \times 30 \times 30 \mathrm{~cm}$,

$\mathrm{b}$ - solenoid that creates a thickened (section) magnetic tube,

$\mathrm{c}$ - discharge condenser,

d - 'belt" plasma yield.

The chamber is filled with a mixture of gases that are mainly low active in a chemical relation, but can be comparatively easily ionized. The approximate composition of mixture: nitrogen $\left(\mathrm{N}_{2}\right)$, gaseous sulphur c.silica and carbon compounds Metalized dispersive inclusions of sodium, lithium, cesium, aluminium and some other elements are possible. Pressure of 1 atm. Voltage in the discharger $\mathbf{3} \div \mathbf{1 0} \boldsymbol{k V}$ in an impulse of an arbitrary duration, but sufficient to ionize the mixture. The magnetic field focuses plasma and directs it in the form of a belt to the MHD-generator; $\boldsymbol{B} \sim \mathbf{0 , 1} \mathbf{- 0 , 3}$ tesla. It is desirable to create a field impulse that will additionally liquefy plasma inside the tube (b). Plasma temperature must not exceed 20 thousand degress.

Therefore, this demonstrates the possibility of converting the energy of quantum plasma condensate to electric.

As it is known, scientists think that the population of the Earth will die not from nuclear weapons, but from waste. $97 \%$ of what is extracted from the earth is in an intermediate state or buried. The world is in such a condition when the harm caused by technology to the environment has exceeded the possibilities of our planet.

Waste can literally be used as a material for producing quantum plasma condensate as a fuel: wastes of industrial plants, dump sites of mine development, waste landfills etc. It is not inconceivable that the distinctive feature of the century XXI civilization will consist in this possibility (using wastes, reappraisal of values, event fight for wastes) Engineering as nature must be rational. There is also another thing that is of utmost importance. The process of energy release is accompanied by phase transformation of a new type of matter. First, matter of a type of plasma liquid is generated from plasma discharge, and then as it is cooled to produce a solid conglomerate of crystalline formation. This type of transformations can be encountered in natural conditions, for example, during volcanic eruptions. It appears that solid plasma phase is a substance which have new unpredictable properties. In any case, during "combustion" we obtain new, probably, verity useful materials as an output. This area can be called electric discharge plasma metallurgy.

Therefore, the authors have developed an entirely new direction in the theory and technology of non-ideal plasma. Conducted research has helped predict and then prove experimentally in laboratory conditions the existence of an entirely new state of matter - quantum plasma condensate combining properties typical of a normal liquid 
(liquidity, surface tension, internal correlations) and those of ionized plasma in the usual sense. The theory developed in our research based on the direct solution of the Schrodinger equation and applicable to the class of states of a continuous spectrum - the perturbation theory considering exchange force - have allowed explaining the observed specific features of plasma phase - quantum plasma condensate, which may and must be used by modern science and technology.

The described research has predicted and proved the existence of an entirely new, alternative renewable, harmless and efficient source of energy on planet Earth - quantum plasma condensate. This source is the only environmentally safe source that does not destroy and pollute the plant; On the contrary, its use cleans the environment of planet Earth.

Mastering the physics of the fifth state of matter -quantum plasma condensate discovered by the authors of this paper will allow focusing on solving the task of cold fusion (including without using external sources of energy)

Therefore, the task of creating new nature-like technologies which do not harm the environment, and exist in harmony with it and allows restoring the disrupted balance between the biosphere and technosphere can be considered as having been solved.

\section{References:-}

1. Kulakov, A.V Spontaneous magnetization of quantum origin plasma / A.V. Kulakov, A.A.Rumyantsev // Technical Physics Journals. - 1988. - T.58, Issue 4. - C.657-660.

2. Kulakov, A.V. Quantum exchange forces in condensed media /A.V.Kulakov, E.V.Orlenko, A.A. Rumayntsev//Moscow, Science.1990.

3. Kulakov, A.V. Experimental proof of the fact of the existence of quantum plasma condensate / A.V.Kulakov, V.V.Rantsev-Kartinov // Bulletin Russian Academy of Sciences Energy. - 2015. - No.1.

4. Kulakov, A.V. Ball lightning as quantum condensate / A.V.Kulakov, A.A. Rumyantsev// Reports of the Academy of Sciences of the USSR. Physics.-1991-T.320, No. 5 - p.1103-1106.

5. Kulakov, A.V Generation of particles of MHD high energy by shock turbulence / A.V.Kulakov, A.A.Rumyantsev // Technical Physics Journals. - 1979. -T. 49-Edition 10-p. 2127-2132.

6. Kulakov, A. V. New Approach to the Plasma Quantum Condensate as a New State of Matter / A. V. Kulakov V. M. Tyutuynnik // International Journal of Current Research. -2017.-Vol.9,№ 3.-P. 47699-47703.

7. Kulakov, A. V. Quantum non-ideal plasma as a source of heat energy. Plasma fuel combustion //International Journal of Current Research. -2017.- Vol.9,№ 6.-P.53361-53365.

8. Kulakov, A.V. Quantum Plasma Condensate as a New Source of Energy: the Operation Principle of the Plasma Ultraviolet Laser/ A V. Kulakov, V. M. Tyutyunnik // Asian Journal of Science and Technology .2017.-Vol.8, Issue 7.-P.5065-5068.

9. Rosa R. Magnetohydrodynamic conversion of energy, M,: Mir, 1970.

10. Satton G. and Sherman A. Fundamentals of magnetohydrodynamic engineering, M.: Mir, 1968, 